\title{
An Achievability Result for Random Networks
}

\author{
Radhika Gowaikar \\ California Institute of Technology \\ Email: gowaikar@caltech.edu
}

\author{
Bertrand Hochwald \\ Bell Laboratories \\ Email: hochwald@lucent.com
}

\author{
Babak Hassibi \\ California Institute of Technology \\ Email: hassibi@caltech.edu
}

\begin{abstract}
We analyze a network of nodes in which pairs communicate over a shared wireless medium. We are interested in the maximum total aggregate traffic flow that is possible through the network. Our model differs substantially from the many existing approaches in that the channel connections in our network are entirely random: we assume that, rather than being governed by geometry and a decay law, the strength of the connections between nodes is drawn independently from a common distribution. Such a model is appropriate for environments where the first order effect that governs the signal strength at a receiving node is a random event (such as the existence of an obstacle), rather than the distance from the transmitter.

We show that the aggregate traffic flow is a strong function of the channel distribution. In particular, we show that for certain distributions, the aggregate traffic flow scales at least as $\frac{n}{(\log n)^{v}}$ for some fixed $v>0$, which is significantly larger than the $O(\sqrt{n})$ results obtained for many geometric models.
\end{abstract}

\section{INTRODUCTION}

Sensory and ad hoc networks have recently attracted much attention in the research community. An early study of such networks appears in the seminal work of Gupta and Kumar [10]. They show that in a grid network of $n$ nodes on the plane having a deterministic power scaling law, less than $\sqrt{n} / 2$ transmitters can talk simultaneously to an average receiver. Similar results for networks with randomly placed nodes can also be obtained (see, for example, [9] for a recent account). Different models can yield somewhat different conclusions [1], [3], [5], [8], [11], [13], [14]; nevertheless, if we do not permit the transmitter/receiver pairs to approach one another [6], the model of a power decay law (as a function of distance) seems to yield a network with less-than-linear growth in the number of nodes that can talk simultaneously. We change the model of the wireless medium from a model based on distance to one based on randomness.

From the study of multi-antenna links [4], [12], it is now generally believed that a rich scattering environment, once thought to be detrimental to wireless communications, may actually be beneficial. We show that a similar effect may hold for the expected aggregate data traffic in a wireless network.

Random models may be preferred over distance-based ones since decay laws of the form $1 / r^{\alpha}$ are usually valid in farfield approximations and may not hold for networks of small physical size that are designed with minimum and maximum distances in mind. Additionally, automatic gain control can mitigate many distance effects. Thus, important signal-strength effects are often due to random fluctuations in the medium. Such models for wireless networks have recently been gaining traction. For example, [7] uses a "radio model" to show that in the presence of obstructions and irregularities, channels become approximately uncorrelated with one another, and the probability of good links between nodes that are far apart increases in wireless local area networks (WLANs).
We adopt the premise that randomness can have a firstorder effect on the behavior of a network. Our model covers environments where the first order effect that governs the signal strength at a receiving node is a random event (such as the existence of an obstacle), rather than the distance from the transmitter. In this sense, it is significantly different from most existing connectivity studies that are based on geometry. We believe that the study of such random networks is important for two reasons: first, many real wireless networks have a substantial and sometimes dominant random component; second, we show that random networks may have qualitatively different scaling laws from the standard $O(\sqrt{n})$ results obtained in geometric models, where $n$ is the number of nodes in the network.

\section{A. Approach}

We summarize our approach. We suppose that the connection strengths between nodes are drawn independently and identically from a given arbitrary distribution. Since we have no "geometric" notion of near neighbors, we introduce the notion of "good" connections. These are connections stronger than a chosen threshold $\beta$. Transmissions to relays and destinations are performed along only good paths. By figuratively drawing a graph whose vertices are all the nodes in the network, yet whose edges are only the good paths, we obtain a specific well-studied random graph model. We leverage known results regarding this to establish disjoint routes between source-destination pairs. Finally, we account for interference between all nodes, including those that do not have "good" connections between them. We demonstrate an achievable throughput as a function of $\beta$. This can be maximized by choosing $\beta$ judiciously.

\section{Model of Transmitted And Received Signals}

We model the wireless network as having narrowband flatfading connections whose powers are independent and identically distributed (i.i.d.) according to an arbitrary distribution $f(\cdot)$. Thus, if $h_{i, j}$ is the connection between nodes $i$ and $j$, then the $\gamma_{i, j}=\left|h_{i, j}\right|^{2}$ are i.i.d. random variables with marginal distribution $f\left(\gamma_{i, j}\right)$. For maximum generality, we allow $f(\gamma)=f_{n}(\gamma)$ to be a function of the number of nodes $n$. As an example, consider:

$$
f(\gamma)=\left(1-p_{n}\right) \cdot \delta(\gamma)+p_{n} \cdot \delta(\gamma-1)
$$

where $\delta(\cdot)$ is the Dirac delta-function. This distribution is a simple model of a shadow-fading environment where, for any pair of nodes, with probability $p_{n}$ there exists a good connection between them (fading causes no loss), and with probability $1-p_{n}$ there exists an obstruction (fading causes a complete loss). 
At the extreme of $p_{n}=0$ all nodes are fully connected, yet very few pairs of nodes can talk simultaneously since the interference dominates. For $p_{n}=1$, everyone is in deep fade and no nodes can talk at all. (We assume a transmission power limit.) Thus we have competing effects as a function of $p_{n}$, and are led to ask: what $p_{n}$ is optimal? What is the resulting network aggregate traffic? Is this optimal $p_{n}$ likely to be something we encounter naturally? If not, can we artificially induce the optimal $p_{n}$ ? More generally, we look at how an arbitrary $f_{n}(\gamma)$ affects the traffic.

\section{A. Detailed model}

Let the network have $n$ nodes labeled $1, \ldots, n$. Every pair of nodes $\{i, j\}(i \neq j)$ is connected by a channel, denoted by the random variable $h_{i, j}=h_{j, i}$. We assume that the channel strengths, $\gamma_{i, j}=\left|h_{i, j}\right|^{2}$ are drawn i.i.d. according to some probability density function (pdf) $f_{n}(\gamma)$. Once drawn, these channel variables do not change with time.

Node $i$ wishes to transmit signal $x_{i}$. We assume that $x_{i}$ is a complex Gaussian random variable with zero mean and unit variance. Each node is permitted a maximum power of $P$ watts.

We incorporate interference and additive noise in our model as follows. Assume that $k$ nodes $i_{1}, i_{2}, \ldots, i_{k}$ are simultaneously transmitting signals $x_{i_{1}}, x_{i_{2}}, \ldots, x_{i_{k}}$ respectively. Then, the signal received by node $j\left(\neq i_{1}, \ldots, i_{k}\right)$ is given by

$$
y_{j}=\sum_{t=1}^{k} \sqrt{P} h_{i_{t}, j} x_{i_{t}}+w_{j}
$$

where $w_{j}$ represents additive noise. The additive noise variables $w_{1}, \ldots, w_{n}$ are i.i.d., drawn from a complex Gaussian distribution of zero mean and variance $\sigma^{2}\left(w_{j} \sim \mathcal{C N}\left(0, \sigma^{2}\right)\right)$. The noise is statistically independent of $x_{i}$.

\section{B. Successful communication}

In equation (2), suppose that only node $i_{1}$ wishes to communicate with node $j$ and the signals $x_{i_{2}}, \ldots, x_{i_{k}}$ are interference. Then the signal-to-interference-plus-noise ratio (SINR) for node $j$ is given by

$$
\rho_{j}=\frac{P \gamma_{i_{1}, j}}{\sigma^{2}+P \sum_{l=2}^{k} \gamma_{i_{l}, j}}
$$

We assume that transmission is successful if and only if the SINR exceeds some threshold $\rho_{0}$.

\section{Network Operation AND OBJective}

We suppose that $k$ nodes, denoted as $s_{1}, \ldots s_{k}$, are randomly chosen as sources. For every $s_{i}$, a destination node $d_{i}$ is chosen at random, thus making $k$ source-destination pairs. We assume that these $2 k$ nodes are all distinct and therefore $k \leq n / 2$. Source $s_{i}$ wishes to transmit message $M_{i}$ to destination $d_{i}$ and has encoded it as signal $x_{i}$.

\section{A. Communicating with Hops}

In general, we suppose that the source-destination pair $\left(s_{i}, d_{i}\right)$ communicates using a sequence of relay nodes $r_{i, 1}, r_{i, 2}, \ldots, r_{i, h-1}$. ( $h=1,2, \ldots$ represents the number of hops.) Define $r_{i, 0}=s_{i}$ and $r_{i, h}=d_{i}$. The path from $s_{i}$ to $d_{i}$ is then $r_{i, 0}=s_{i}, r_{i, 1}, \ldots, r_{i, h-1}, r_{i, h}=d_{i}$. In time slot $t+1$ we have nodes $r_{1, t}, \ldots, r_{k, t}$ transmitting simultaneously to nodes $r_{1, t+1}, \ldots, r_{k, t+1}$ respectively. We ask that nodes $r_{1, t+1}, \ldots, r_{k, t+1}$ decode their respective signals $x_{1}, \ldots, x_{k}$ and transmit them to the next set of relay nodes in the $(t+2)$ th time slot, and so on. A natural condition to impose is that the relay nodes that are receiving (or transmitting) messages in any time slot be distinct, i.e., the messages do not collide. In addition, we ask that relay nodes not receive and transmit at the same time. We refer to these conditions together as the property of no collisions in the rest of the paper.

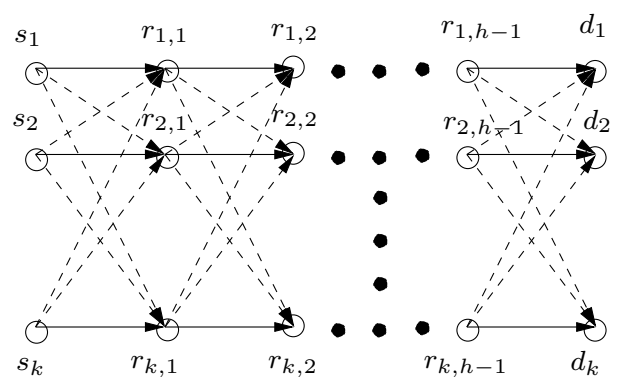

Fig. 1. Schedule of relay nodes: Source $s_{i}$ communicates with destination $d_{i}$ using relays $r_{i, 1}, \ldots, r_{i, h-1}$. The solid lines indicate intended transmissions and the dashed lines indicate potential interference. The conditions on a schedule are that no node have to receive or transmit more than one message in any time slot and that no node have to perform transmission and reception simultaneously.

\section{B. Throughput}

With the above procedure, we have $k$ simultaneous communications occurring in $h$ time slots. Message $M_{i}$ reaches the intended destination $d_{i}$ successfully if it can be decoded by each relay $r_{i, t}$. Assume that a fraction $1-\epsilon$ of messages reach their intended destinations in this way. Then, we define the throughput as

$$
T=(1-\epsilon) \frac{k}{h} \log \left(1+\rho_{0}\right)
$$

The number of source-destination pairs $k$, the fraction of dropped messages $\epsilon$, the SINR threshold $\rho_{0}$ and hence the throughput $T$ depend on $n$ and we sometimes denote them by $k_{n}, \epsilon_{n}, \rho_{0, n}$ and $T_{n}$. Typically, we force $\epsilon_{n}$ to go to zero. We demonstrate a scheme for choosing the relay nodes and analyze the throughput as well as the performance for this scheme. Thus, we give an achievability result for $T_{n}$. We begin by stating this result.

\section{MAin Result}

Theorem 1: Consider a network on $n$ nodes whose edge strengths are drawn i.i.d. from a probability distribution function $f_{n}(\gamma)$. Let $F_{n}(\gamma)$ denote the cumulative distribution function corresponding to $f_{n}(\gamma)$ and define $Q_{n}(\gamma)=1-$ $F_{n}(\gamma)$. Choose any $\beta_{n}$ such that $Q_{n}\left(\beta_{n}\right)=\frac{\log n+\omega_{n}}{n}$, where $\omega_{n} \rightarrow \infty$ as $n \rightarrow \infty$. Then there exists a constant $\alpha$ such that, for $n \rightarrow \infty$, a throughput of

$$
T=\left(1-\epsilon_{n}\right) \alpha k_{n}\left(\beta_{n}\right) \frac{\log \left(n Q_{n}\left(\beta_{n}\right)\right)}{\log n} \log \left(1+\frac{a \beta_{n}}{\frac{\sigma^{2}}{P}+k_{n}\left(\beta_{n}\right) \mu_{\gamma}}\right)
$$

is achievable where $k_{n}\left(\beta_{n}\right)$ is the maximum quantity satisfying the two conditions:

$$
k_{n}\left(\beta_{n}\right) \leq \alpha n \frac{\log \left(n Q_{n}\left(\beta_{n}\right)\right)}{\log n}
$$




$$
\epsilon_{n} \leq \frac{a^{2}}{\alpha(1-a)^{2}} \frac{k_{n}\left(\beta_{n}\right) \sigma_{\gamma}^{2}}{\left(\frac{\sigma^{2}}{P}+k_{n}\left(\beta_{n}\right) \mu_{\gamma}\right)^{2}} \frac{\log n}{\log \left(n Q_{n}\left(\beta_{n}\right)\right)} \rightarrow 0
$$

where $\mu_{\gamma}$ and $\sigma_{\gamma}^{2}$ are the mean and variance of $\gamma$ respectively. The SINR threshold is $\rho_{0}=\frac{a \beta_{n}}{\frac{\sigma^{2}}{P}+k_{n}\left(\beta_{n}\right) \mu_{\gamma}}$ where $a$ is any constant less than 1.

The parameter $\beta_{n}$ satisfying $Q_{n}\left(\beta_{n}\right)=\frac{\log n+\omega_{n}}{n}$ is the threshold mentioned in Section I-A that allows us to introduce a random graph model. Condition (5) is needed to ensure that we may obtain a non-colliding schedule in this random graph. (See Section V.) Once the schedule is obtained, we incorporate the effects of interference between non-colliding transmissions and analyze the error, $\epsilon_{n}$, in Section VI. Condition (6) forces $\epsilon_{n}$ to go to zero. In Section VII we combine the results of Sections V and VI to prove the theorem. We show how to apply the theorem and choose $\beta_{n}$ in Section VIII where we give several examples.

\section{Scheduling TRANSmissions}

With a view to meeting a minimum SINR of $\rho_{0}$ at every relay node at every hop, we impose the condition that each transmitting link be stronger than some threshold $\beta_{n}$. We require that $\gamma_{r_{i, t}, r_{i, t+1}} \geq \beta_{n}$, where $\beta_{n}$ is a design parameter. We call links that satisfy $\gamma_{i, j} \geq \beta_{n}$ as good. We require the path from $s_{i}$ to $d_{i}$ to use only good links.

By making $\beta_{n}$ large we increase the quality of the link. However, if we make it too large we risk not being able to form an uninterrupted path of good links from the source to the destination. In this section, we determine the relation between $\beta_{n}$ and the lengths of source-destination paths.

Define $p_{n}=\mathrm{P}\left(\gamma \geq \beta_{n}\right)$ (for convenience, we drop the subscript $n$ in the rest of this section). Using our wireless communication network, we define a graph on $n$ vertices as follows: For (distinct) vertices $i$ and $j$ of the graph, draw an edge $(i, j)$ if and only if $\gamma_{i, j} \geq \beta_{n}$ in the network. Call the resulting graph $G(n, p)$. The graph $G(n, p)$ then becomes an instance of a model called $\mathcal{G}(n, p)$ on $n$ vertices in which edges are chosen independently and with probability $p$ [2]. This graph shows the possible paths from $s_{i}$ to $d_{i}$ using only good links, but does not show the interference between paths. We examine this interference in Section VI.

Graphs taken from the model $\mathcal{G}(n, p)$ have many known properties regarding their connectivity, maximum minimum distance etc. [2], [15]. We invoke a relatively recent result regarding vertex-disjoint paths for this model.

\section{A. Scheduling using vertex-disjoint paths in $G(n, p)$}

Two paths that do not share a vertex are called vertexdisjoint. Note that any two paths that are vertex-disjoint satisfy our "no-collisions" property; however, the reverse statement is not true. Thus, the vertex-disjoint condition is stronger than our requirement of non-colliding paths. For a set of $k$ (disjoint) pairs of vertices $\left(s_{i}, d_{i}\right)$, the question of whether there exists a set of vertex-disjoint paths connecting them is addressed in [16]. Their result states that, under certain randomness conditions, with high probability, for every set of $k$ pairs $\left(s_{i}, d_{i}\right)$ and $k$ not greater than $\alpha n \frac{\log n p}{\log n}$, where $\alpha$ is a constant, there exists a set of vertex-disjoint paths. It turns out that the randomness conditions required for their result are easily met in our network setup. Here we state a simplified version of their result that can be directly used for our purposes.

Theorem 2: Suppose that $G=G(n, p)$ and $p \geq \frac{\log n+\omega_{n}}{n}$, where $\omega_{n} \rightarrow \infty$. Then there exists a constant $\alpha>0$ such that, with probability approaching 1 , there are vertex-disjoint paths connecting $s_{i}$ to $d_{i}$ for any set of disjoint, randomly chosen source-destination pairs

$$
F=\left\{\left(s_{i}, d_{i}\right) \mid s_{i}, d_{i} \in\{1, \ldots, n\}, i=1, \ldots, k\right\}
$$

provided $k=|F|$ is not greater than $\alpha n \frac{\log n p}{\log n}$.

The constant $\alpha$ in this theorem is the same $\alpha$ required in Theorem 1. It is not explicitly specified. It is now easy to reach a conclusion regarding the lengths that these $k$ paths can have. We state it without proof in the following lemma.

Lemma 1: Almost all of the $k=\alpha n \frac{\log n p}{\log n}$ vertex-disjoint paths obtainable under Theorem 2 have lengths that grow no faster than $\frac{\log n}{\alpha \log n p}$.

Hence the number of hops $h$ is (asymptotically) at most $\frac{\log n}{\alpha \log n p}$. We use this fact in the error analysis in the following section.

\section{Probability of ERror}

Algorithms that choose non-colliding paths without using information regarding the edges between vertices along one path to vertices along another have the property that these edges are i.i.d. Bernoulli distributed with parameter $p$. An example of a randomized algorithm that does this can be found in [16]. From this we conclude that the channel connections between nodes along different paths in the network are i.i.d. with distribution $f_{n}(\gamma)$.

We now consider the probability that a particular message fails to reach its intended destination. Destination $d_{i}$ fails to receive message $M_{i}$ if the SINR falls below $\rho_{0}$ at any of the $h$ relay nodes $r_{i, 1}, \ldots, r_{i, h}=d_{i}$. Denote by $E_{t}$ the event that relay node $r_{i, t}$ does have an SINR greater than $\rho_{0}$. Note that the events $E_{1}, \ldots, E_{h}$ are identical. Therefore we have,

$$
\begin{aligned}
& \mathrm{P}\left(M_{i} \text { is received successfully }\right) \\
= & \mathrm{P}\left(\bigcap_{t=1}^{h} E_{t}\right)=1-\mathrm{P}\left(\bigcup_{t=1}^{h} \sim E_{t}\right) \geq 1-h \mathrm{P}\left(\sim E_{1}\right)
\end{aligned}
$$

where the inequality comes from the union bound. We now compute $\mathrm{P}\left(\sim E_{1}\right)$. This is the event that node $r_{i, 1}$ has an SINR lower than $\rho_{0}$.

$$
\begin{aligned}
& \mathrm{P}\left(\sim E_{1}\right) \\
= & \mathrm{P}\left(\rho_{r_{i, 1}} \leq \rho_{0}\right) \\
= & \mathrm{P}\left(\frac{P \gamma_{s_{i}, r_{i, 1}}}{\sigma^{2}+P \sum_{j \neq i} \gamma_{s_{j}, r_{i, 1}}} \leq \rho_{0}\right) \\
\leq & \mathrm{P}\left(\frac{1}{k-1} \sum_{j \neq i} \gamma_{s_{j}, r_{i, 1}}-\mu_{\gamma} \geq \frac{P \beta_{n}-\rho_{0} \sigma^{2}}{(k-1) P \rho_{0}}-\mu_{\gamma}\right) \\
\leq & \mathrm{P}\left(\left|\frac{1}{k-1} \sum_{j \neq i} \gamma_{s_{j}, r_{i, 1}}-\mu_{\gamma}\right| \geq \frac{P \beta_{n}-\rho_{0} \sigma^{2}}{(k-1) P \rho_{0}}-\mu_{\gamma}\right) \\
\leq & \frac{\sigma_{\gamma}^{2} /(k-1)}{\left(\frac{P \beta_{n}-\rho_{0} \sigma^{2}}{(k-1) P \rho_{0}}-\mu_{\gamma}\right)^{2}}
\end{aligned}
$$


where the first inequality comes from rearranging terms and because $\gamma_{s_{i}, r_{i, 1}} \geq \beta_{n}$ and (7) comes from the Chebyshev inequality and the fact that the variance of $\frac{1}{k-1} \sum_{j \neq i} \gamma_{s_{j}, r_{i, 1}}$ is $\sigma_{\gamma}^{2} /(k-1)$. The second inequality requires the condition $\frac{P \beta_{n}-\rho_{0} \sigma^{2}}{(k-1) P \rho_{0}}-\mu_{\gamma} \geq 0$, or

$$
\rho_{0} \leq \frac{\beta_{n}}{\frac{\sigma^{2}}{P}+(k-1) \mu_{\gamma}} .
$$

The probability of error in the communication of message $M_{i}$, or $\epsilon_{n}$, is no greater than $h P\left(\sim E_{1}\right)$. Note that this is the same for all the messages. From Lemma 1 and the above, we have

$$
\epsilon_{n} \leq h \mathrm{P}\left(\sim E_{1}\right) \leq \frac{\log n}{\alpha \log n p} \frac{\sigma_{\gamma}^{2}}{(k-1)\left(\frac{P \beta_{n}-\rho_{0} \sigma^{2}}{(k-1) P \rho_{0}}-\mu_{\gamma}\right)^{2}}
$$

We force the last expression to go to zero.

\section{Proof of Theorem 1}

We have the condition $k \leq \alpha n \frac{\log n p}{\log n}$ from Theorem 2 (this gives us (5)), the condition in (8) and the condition that the upperbound on $\epsilon_{n}$ from (9) go to zero. With these we need to maximize the throughput. For a fixed $\beta_{n}$ (and hence fixed $p$ and $\left.h=\frac{\log n}{\alpha \log n p}\right)$ and with $\epsilon_{n} \rightarrow 0$, the $\rho_{0}$ that maximizes $T=\left(1-\epsilon_{n}\right) \frac{k}{h} \log \left(1+\rho_{0}\right)$ is the largest that satisfies (8). This gives us $\rho_{0}=\frac{a \beta_{n}}{\frac{\sigma^{2}}{P}+(k-1) \mu_{\gamma}}$ where $a<1$ is a constant. Plugging this in (9) gives us (6). Thus, any $k$ that satisfies (5) and (6) is permissible and gives us an achievable throughput of the form (4). In particular if we choose the maximum permissible $k$ we get an achievable throughput. This gives us Theorem 1. Ideally, we should find the optimum $k$ that maximizes the throughput of (4). Often, the maximum permissible $k$ turns out to be optimal. We also remark that a further optimization over all $\beta_{n}$ that satisfy $Q_{n}\left(\beta_{n}\right)=\frac{\log n+\omega_{n}}{n}$ can give the maximum achievable throughput. For more details, see [17].

\section{EXAMPLES AND Simulations}

In this section we apply Theorem 1 to some particular channel distributions and obtain achievable throughputs.

\section{A. Shadow fading model}

We revisit the model of equation (1). This pdf models the situation where strong shadow fading is present. The signal power is 0 in the presence of an obstruction and is 1 otherwise. We find the value of $p_{n}$ that maximizes the throughput. A natural choice for $\beta_{n}$ is 1 which gives $Q_{n}\left(\beta_{n}\right)=p_{n}$. We need to satisfy $p_{n} \geq\left(\log n+\omega_{n}\right) / n$ (where $\omega_{n} \rightarrow \infty$ ) in order to use Theorem 1. It turns out that the smallest permissible value of $p_{n}$ is optimal. We get the following result.

Corollary 1: Consider a network on $n$ nodes where edge strengths are drawn i.i.d. from the distribution in (1). Then, for large $n$, the throughput is maximized for $p_{n}=\frac{\log n+\omega_{n}}{n}$ and is given by

$$
T \approx \frac{\alpha n}{w_{n}} \frac{\log ^{2}(\log n)}{\log ^{3} n} \log \left(1+\frac{a P}{\sigma^{2}}\right) .
$$

where $w_{n}$ and $\omega_{n}$ are any functions going to infinity and $a<$ $1, \alpha<1$ are constants.

This throughput is almost linear in $n$ and requires the network to be sparsely connected; each node is connected with only approximately $\log n$ other nodes. Interestingly, increasing or decreasing this connectivity has a detrimental effect.

\section{B. An exponential density}

Let $f_{n}(\gamma)=e^{-\gamma}$. The parameters that maximize the throughput turn out to be $\beta_{n}=\log n / 2$, which gives $h=\frac{1}{2 \alpha}$, $k=\alpha n / 2$ and $\rho_{0}=\log n / \alpha n$. With this $\epsilon_{n}=\frac{a^{2}}{\alpha^{2}(1-a)^{2}} \frac{4}{n} \rightarrow$ 0 . This gives the following result.

Corollary 2: Consider a network on $n$ nodes where edge strengths are drawn i.i.d. from a distribution $f_{n}(\gamma)=e^{-\gamma}$. Then a throughput of

$$
T=\frac{a \alpha \log n}{4}
$$

is achievable where $\alpha<1, a<1$ are constants.

We see that a random network dominated by an exponential pdf has a throughput that scales only logarithmically with $n$. This network has good connectivity since the number of hops is constant, but unfortunately is also dominated by interference.

\section{Density obtained from a decay law}

Suppose that we are working with a network in which nodes are randomly placed at lattice points with edge distance $d$ in a circular arrangement. Assume that the density of nodes is fixed as $\Delta$. Assume that a power decay law of $1 / r^{m}, m>0$ holds, where $r$ is the distance. When a node at the center of this disk transmits with power $P=1$, the marginal distribution of the signal powers received by other nodes is given by

$f_{n}(\gamma)=\frac{4 \pi \Delta}{n m} \frac{1}{\gamma^{1+\frac{2}{m}}}, \gamma \in\left[\left(\frac{2 \pi \Delta}{n+2 \pi \Delta d^{2}}\right)^{m / 2}, \frac{1}{d^{m}}\right], m>0$.

Because of the geometry of the network the joint distribution is not simply the product of the marginals. However, we assume that the channel strengths are drawn i.i.d. from (10). Applying Therorem 1 to this gives the following results.

Corollary 3: Consider a network on $n$ nodes where edge strengths are drawn i.i.d. from the distribution in (10). Then the achievable throughput is given by

$$
T= \begin{cases}\left(1-\epsilon_{n}\right) \frac{a(2-m) \alpha}{2} \frac{\log \left(\log n+\omega_{n}\right)}{\log n\left(\log n+\omega_{n}\right)^{m / 2}} n^{m / 2} & m<2 \\ \left(1-\epsilon_{n}\right) a \alpha \frac{\log \left(\log n+\omega_{n}\right)}{\log ^{2} n\left(\log n+\omega_{n}\right)} n & m=2 \\ \left(1-\epsilon_{n}\right) \frac{P a \alpha(2 \pi \Delta)^{m / 2}}{\sigma^{2} w_{n}} \frac{\log ^{2}\left(\log n+\omega_{n}\right)}{\log ^{2} n\left(\log n+\omega_{n}\right)^{m / 2}} n & m>2 .\end{cases}
$$

where $w_{n}$ and $\omega_{n}$ are any functions going to infinity, $a<1$, $\alpha<1$ are constants and $\epsilon_{n} \rightarrow 0$ in every case.

We see that almost linear throughput can be obtained for $m \geq 2$. This differs substantially from the $O(\sqrt{n})$ results obtained for the structured deterministic model with the same decay law. Our results show that it is not the marginal distribution of the power that impedes the throughput in a geometric power-decay network, but rather the spatial distribution of these powers.

\section{A heavy tail distribution}

We state the result for a simple heavy-tail distribution.

Corollary 4: Consider a network on $n$ nodes where edge strengths are drawn i.i.d. from the distribution $f_{n}(\gamma)=$ $\frac{c}{1+\gamma^{4}}, \gamma \geq 0$. The throughput is then given by

$$
T \approx \frac{a(c / 3)^{1 / 3} \alpha}{\mu_{\gamma}} \frac{\log \log n}{\log ^{4 / 3} n} n^{1 / 3} .
$$

where $a<1, \alpha<1$ are constants. 


\section{E. Simulations}

Figure 2 shows the aggregate throughput curves for the shadow-fading network of Section VIII-A (upper curve) and the decay density network of Section VIII-C (lower curve). Simulations for networks from 100 to 1200 nodes are done with $P=1$ and $\sigma^{2}=0.1$. For the shadow-fading network $p=\frac{2 \log n}{n}$ is used and for the decay law density the parameters used are $d=1, \Delta=1$, and $m=3$. In both cases, the throughput increases almost linearly (with different slopes) as expected from the analysis presented earlier. The decaydensity network has a lower aggregate throughput, which is not surprising since it suffers from more interference than the shadow-fading network. Further simulation details can be found in [17].

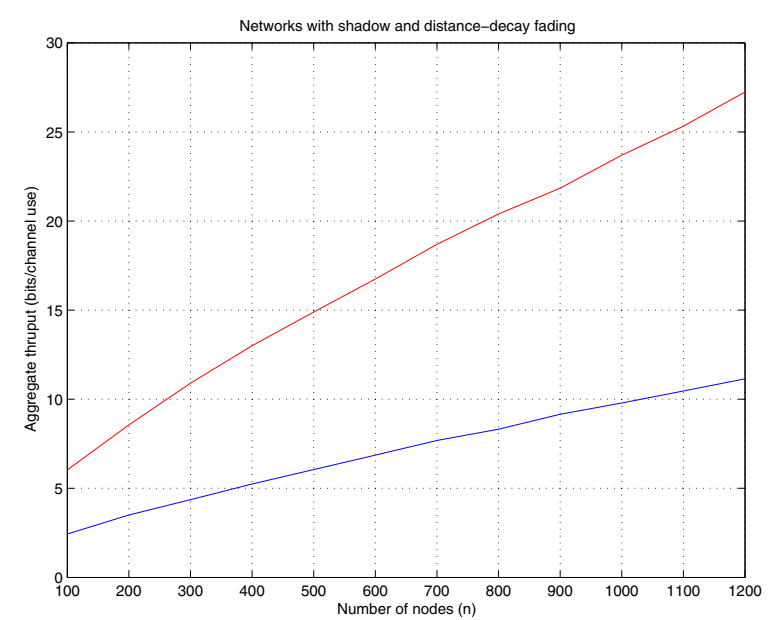

Fig. 2. Aggregate throughput versus number of nodes $n$ in the shadow-fading network of Section VIII-A (upper curve) and in the decay-density network of Section VIII-C (lower curve).

\section{CONCLUSION}

We have presented a new model for ad hoc networks that has links which are randomly drawn from a specific distribution. We have devised a method of operating this network using relays and shown that for certain distributions, such operation gives us an achievable throughput of $n /(\log n)^{v}$ for some fixed $v>0$. In particular, distributions that have a certain sparsity of "good" connections seem to perform the best. This is encouraging and of possible use in the design of obstacle placement or density in ad hoc networks.

Finding decentralized schemes for scheduling of relay nodes and proving upper bounds on the achievable throughput are possible directions for future work, as is the study of networks models that lie somewhere in between the i.i.d. random networks described here and the geometry-based ones prevalent in the literature.

\section{ACKNOWLEDGMENT}

This work is supported in part by the National Science Foundation under grant nos. CCR-0133818 and CCR0326554, by the David and Lucille Packard Foundation, and by Caltech's Lee Center for Advanced Networking.

\section{REFERENCES}

[1] F. Baccelli, M. Klein, M. Lebourges and S. Zuyev, "Stochastic geometry and architecture of communication networks," J. Telecommunication Systems, vol. 7, pp. 209-227, 1997.

[2] B. Bollobás, Random Graphs, 2nd ed., Cambridge: University Press, 2001

[3] O. Dousse and P. Thiran, "Connectivity versus capacity in dense ad hoc networks," Proc. 23rd INFOCOM, Hong Kong, Mar. 2004.

[4] G. J. Foschini, "Layered space-time architecture for wireless communication in a fading environment when using multi-element antennas," Bell Labs. Tech. J., vol. 1, no. 2, pp. 41-59, 1996.

[5] M. Gastpar and M. Vetterli, "On the capacity of wireless networks: the relay case," Proc. 21st INFOCOM, New York, Jun. 2002, pp. 1577-1586.

[6] M. Grossglauser and D. Tse, "Mobility increases the capacity of ad-hoc wireless networks," IEEE/ACM Trans. on Networking, vol. 10, pp. 477486, Aug. 2002.

[7] R. Hekmat and P. van Mieghem, "Study of connectivity in wireless ad-hoc networks with an improved radio model," Proc. 2nd Workshop on Model. and Optim. in Mobile, Ad Hoc and Wireless Networks, Cambridge, UK, Mar. 2004.

[8] O. Léveque and E. Telatar, "Information theoretic upper bounds on the capacity of large extended ad hoc wireless networks," to appear in the IEEE Trans. on Info. Theory.

[9] M. Franceschetti, O. Dousse, D. Tse and P. Thiran, "On the throughput capacity of random wireless networks," submitted to IEEE Trans. Info. Theory, 2004.

[10] P. Gupta and P. R. Kumar "The capacity of wireless networks," IEEE Trans. Info. Theory, vol. 46, pp. 388-404, Mar. 2000.

[11] P. Gupta and P. R. Kumar, "Towards an information theory of large networks: an achievable rate region," IEEE Trans. Info. Theory, vol. 49, pp. 1877-1894, Aug. 2003.

[12] I. E. Telatar, "Capacity of multi-antenna Gaussian channels," Eur. Trans. Telecom., vol. 10, pp. 585-595, Nov. 1999.

[13] O. Tonguz and G. Ferrari, "Connectivity and transport capacity in ad hoc wireless networks," Proc. 32nd IEEE Comm. Theory Workshop, Mesa, AZ, Apr. 2003

[14] L.-L. Xie and P. R. Kumar, "A network information theory for wireless communication: Scaling laws and optimal operation," IEEE Trans. Info. Theory, vol. 50, pp. 748-767, May 2004.

[15] F. Chung and L. Lu, "The diameter of random sparse graphs," Advances in Appl. Math, vol. 26, pp. 257-279, 2001.

[16] A. Z. Broder, A. M Frieze, S. Suen and E. Upfal, "An Efficient Algorithm for the Vertex-Disjoint Paths Problem in Random Graphs," Proc 7th Symp. Discrete Algorithms, Atlanta, 1996, pp 261- 268.

[17] R. Gowaikar, B. Hochwald and B. Hassibi, "Capacity of Wireless Networks with Random Connections," IEEE Trans. Info. Theory, in preparation. 